\title{
Unravelling the Physical Mechanisms that Determine Microstructural Evolution of Ultrathin Volmer-Weber Films
}

\author{
Viktor Elofsson, Daniel Magnfält, Peter Münger and Kostas Sarakinos
}

\section{Linköping University Post Print}

\section{Tweet}

N.B.: When citing this work, cite the original article.

Original Publication:

Viktor Elofsson, Daniel Magnfält, Peter Münger and Kostas Sarakinos, Unravelling the Physical Mechanisms that Determine Microstructural Evolution of Ultrathin Volmer-Weber Films, 2014, Journal of Applied Physics, (116), 4, 044302.

http://dx.doi.org/10.1063/1.4890522

Copyright: American Institute of Physics (AIP)

http://www.aip.org/

Postprint available at: Linköping University Electronic Press

http://urn.kb.se/resolve?urn=urn:nbn:se:liu:diva-103920 


\title{
Unravelling the Physical Mechanisms that Determine Microstructural Evolution of Ultrathin Volmer-Weber Films
}

\author{
V. Elofsson, ${ }^{1, \text { a) }}$ B. Lü, ${ }^{1}$ D. Magnfält, ${ }^{1}$ E. P. Münger, ${ }^{1}$ and K. Sarakinos ${ }^{1}$ \\ Department of Physics, Chemistry and Biology (IFM), Linköping University, \\ SE-581 83 Linköping, Sweden
}

(Dated: 13 June 2014)

The initial formation stages (i.e., island nucleation, island growth, and island coalescence) set characteristic length scales during growth of thin films from the vapor phase. They are, thus, decisive for morphological and microstructural features of films and nanostructures. Each of the initial formation stages has previously been wellinvestigated separately for the case of Volmer-Weber growth, but knowledge on how and to what extent each stage individually and all together affect the microstructural evolution is still lacking. Here we address this question using growth of $\mathrm{Ag}$ on $\mathrm{SiO}_{2}$ from pulsed vapor fluxes as a case study. By combining in situ growth monitoring, ex situ imaging and growth simulations we systematically study the growth evolution all the way from nucleation to formation of a continuous film and establish the effect of the vapor flux time domain on the scaling behaviour of characteristic growth transitions (elongation transition, percolation and continuous film formation). Our data reveal a pulsing frequency dependence for the characteristic film growth transitions, where the nominal transition thickness decreases with increasing pulsing frequency up to a certain value after which a steady-state behaviour is observed. The scaling behaviour is shown to result from differences in island sizes and densities, as dictated by the initial film formation stages. These differences are determined solely by the interplay between the characteristics of the vapor flux and time required for island coalescence to be completed. In particular, our data provide evidence that the steady-state scaling regime of the characteristic growth transitions is caused by island growth that hinders coalescence from being completed.

PACS numbers: 68.55.at, 68.55.jd, 81.10.Bk, 81.15.Aa

a) Author to whom correspondence should be addressed. E-mail: vikjo@ifm.liu.se 


\section{INTRODUCTION}

Driven by weak film-substrate interaction and substrate surface energies smaller than that of the deposit, Volmer-Weber type thin film growth starts with vapor condensation and nucleation of separated three-dimensional atomic islands on a substrate surface ${ }^{1}$. These islands grow in size, coalesce and eventually form a continuous film. The initial formation stages; island nucleation, growth and coalescence, set the characteristic length scales of the growing film. They are thus decisive for morphological features of films and nanostructures such as island size and separation in the island growth regime as well as surface roughness, and thickness at which conductive (in the case of metallic films) and continuous films are formed $^{2}$. These features, in turn, are important for optical ${ }^{3}$, electrical and magnetic ${ }^{4}$ properties of the films. A fundamental understanding of the role of each of those growth stages is thus paramount to design films and nanostructures in a knowledge-based manner.

Thin film deposition from the vapor phase is a non-equilibrium process in which dynamics and characteristics of each growth stage are mainly dictated by growth kinetics. An illustrative way to study the correlation between growth evolution and kinetics is by establishing the effect of the latter on scaling behaviour of physical quantities that describe and determine growth. For instance, the steady-state island density, $N$, and hence the initial island size and separation, for Volmer-Weber growth is predicted from theory to scale as ${ }^{5}$

$$
N \sim\left(F_{\text {avg }} / D\right)^{2 / 7}
$$

for a critical island size of one atom. In Eq. (1) $F_{\text {avg }}$ is the time-averaged deposition rate and $D$ the adatom diffusivity. The nuclei density can thus be increased by increasing the incident flux or decreasing the diffusivity (e.g., by lowering the growth temperature). $F_{a v g}$ also determines the island growth rate, which together with the island separation, controls the island impingement rate $1 / \tau_{i m p}$ ( $\tau_{i m p}$ is the time between two island impingement events). Upon impingement the atomic islands start to coalesce, in order to reduce the surface free energy by forming a single island. The coalescence completion rate $1 / \tau_{\text {coal }}\left(\tau_{\text {coal }}\right.$ is the time for a coalescence event to be completed) for surface diffusion driven coalescence decreases with island size as ${ }^{6,7}$

$$
1 / \tau_{\text {coal }}=B / R^{4}
$$

where $B$ is a material and temperature dependent coalescence constant and $R$ the radius 
of coalescing islands. As islands grow larger in size there is a point at which $1 / \tau_{\text {imp }}$ becomes larger than $1 / \tau_{\text {coal }}$. This implies that a single island impinges on a coalescing island pair before the coalescence is completed, which in turn leads to elongated island structures separated by voids. The characteristic thickness at which this happens is known as the elongation transition thickness, $d_{\text {elong }}$, and is the first step towards the formation of a continuous film. Results from analytical models and growth simulations have shown that $d_{\text {elong }}$ scales according to the relation ${ }^{8}$

$$
d_{\text {elong }} \sim\left(F_{\text {avg }} / B\right)^{-1 / 3}
$$

As growth continues elongated clusters of islands impinge on each other and form interconnected networks that in the case of a metallic film provide paths for electrical conductivity over macroscopic distances (this point during the growth is referred to as percolation). Subsequent deposition fills in the voids and forms a continuous film.

Additional insight on and control over dynamics of the initial film formation stages can become possible by providing the vapor flux in pulses with well-defined width $\left(t_{o n}\right)$, frequency $(f)$ and amplitude $\left(F_{i}\right)$. These fluxes have implications for film microstructural evolution when their time scale becomes comparable with time scales of surface processes during film growth. Jensen and Niemeyer ${ }^{9}$ studied the effect of pulsed deposition fluxes on $N$ using kinetic Monte Carlo (KMC) simulations and analytical modeling for a critical island size of one atom. They theorized that when the average time, $\tau_{m}$, for an adatom to get incorporated into a stable island (the adatom lifetime) is considerably larger than the pulse period $\left(\tau_{m} \gg 1 / f\right.$, slow diffusivity regime) adatoms do not vanish between successive pulses. Thus, the substrate experiences a continuous vapor flux $F_{a v g}=F_{i} t_{\text {on }} f$ and $N$ scales according to Eq. $(1)^{9}$. On the contrary, when $t_{o n} \gg \tau_{m}$ (fast diffusivity regime) adatom diffusion and island nucleation happen within a single pulse and $N$ scales as ${ }^{9}$

$$
N \sim\left(F_{i} / D\right)^{2 / 7}
$$

An intermediate region separates the two regimes $\left(t_{o n}<\tau_{m}<1 / f\right)$, in which adatoms still diffuse and nucleate after the end of the vapor pulse, but vanish before the next pulse arrives. In this regime the adatom diffusivity is not the rate limiting step for nucleation and the scaling relationship is ${ }^{9}$

$$
N \sim\left(F_{i} t_{o n}\right)^{1 / 2}
$$


Numerous researchers have employed pulsed vapor fluxes characterized by $F_{i} \gg F_{a v g}$ these fluxes have primarily been generated by pulsed laser deposition (PLD) — and found substantial increase of $N$ as compared to continuous fluxes as predicted by Eqs. (1), (4) and $(5)^{10-13}$. Besides nucleation, the effect of pulsed fluxes (generated by PLD) with constant deposition rate per pulse, $F_{p}\left(F_{p}=F_{i} t_{o n}\right)$, on the percolation thickness, $d_{\text {percol }}$, of Ag films grown on $\mathrm{SiO}_{2}$ (an archetype system for Volmer-Weber growth) was studied by Warrender and Aziz ${ }^{14}$. They found that $d_{\text {percol }}$ scales as a power-law with $f$ (or correspondingly $F_{\text {avg }}$ ) and that the scaling exponent takes values from 0 to -0.34 . Combining experimental data with KMC simulations they suggested that the growth scaling behaviour is set by the relation between pulsing period (i.e., temporal separation between vapor pulses) and coalescence time $^{14}$. The importance of other initial growth stages (i.e., island nucleation and growth) was not explicitly investigated and neither was the effect of the initial growth stages on the film microstructural evolution beyond $d_{\text {percol }}$. Moreover, vapor fluxes generated by PLD are inherently highly energetic, with energies up to hundreds of $\mathrm{eV}^{15}$. The interactions of these fluxes with the growing film can trigger surface and subsurface processes, which in turn can alter the characteristics of island nucleation, growth and coalescence ${ }^{16}$. Recently, Magnfält et al. ${ }^{17}$ experimentally generated pulsed fluxes — by means of a plasma based process termed high power impulse magnetron sputtering (HiPIMS) - in a way that the time domain and the energetic bombardment could be decoupled. Using these deposition fluxes they demonstrated that the thickness at which a continuous $\mathrm{Ag}$ film deposited on $\mathrm{SiO}_{2}$ is formed, $d_{\text {cont }}$, scales with $f$ in a way that resembles the behaviour reported by Warrender and Aziz ${ }^{14}$ for $d_{\text {percol }}$. They also estimated $\tau_{m}$ and $\tau_{\text {coal }}$ and suggested that both nucleation and coalescence may be responsible for the dependence of $d_{\text {cont }}$ on $f$.

In this study we unravel key physical mechanisms that determine the effect of initial formation stages on microstructural evolution of thin films that grow in Volmer-Weber fashion. As a case study we grow $\mathrm{Ag}$ on $\mathrm{SiO}_{2}$ using the pulsed vapor fluxes generated by Magnfält et $a l .{ }^{17}$. The growth evolution is then systematically studied all the way from nucleation to the formation of a continuous film by combining in situ growth monitoring, ex situ imaging and growth simulations. This allows us to establish the effect of the vapor flux time domain on the scaling behaviour of characteristic growth transitions (elongation transition, percolation and continuous film formation). Our results show that all characteristic transition thicknesses exhibit the same frequency dependence, i.e., they are found to decrease 
with frequency up to a certain point after which a steady-state behaviour is observed. The scaling behaviour is shown to be set by differences in island sizes and densities as dictated by the initial formation stages. In particular, our findings suggest that the steady-state behaviour is reached when island growth hinders coalescence leading to a coalescence free growth regime.

\section{RESEARCH STRATEGY}

\section{A. Experiments}

\section{Film growth}

Films were grown in a vacuum chamber with a minimum base pressure of $1.3 \cdot 10^{-6}$ Pa from a circular Ag target (purity 99.99\%) with a diameter of $75 \mathrm{~mm}$. Depositions were performed on electrically floating Si (100) substrates covered with a $3000 \AA$ thermally grown $\mathrm{SiO}_{2}$ at an Ar buffer gas (99.9997\% purity) pressure of $0.67 \mathrm{~Pa}$. The substrates were not intentionally heated and in order to minimize any radiative heating from the cathode they were placed at a distance of $12.5 \mathrm{~cm}$ away from the cathode. Furthermore, the cathode was positioned at an angle of $40^{\circ}$ with respect to the substrate normal.

Pulsed deposition fluxes were generated by the deposition technique high power impulse

magnetron sputtering (HiPIMS) ${ }^{18-24}$. Power pulses with a width of $50 \mu$ s and an energy per pulse of $20 \mathrm{~mJ}$ were used for various pulsing frequencies in the range $50-1000 \mathrm{~Hz}$ to probe the effect of flux time domain on film growth. This pulse configuration leads to a deposition flux with low energies (up to $\sim 15 \mathrm{eV})$ and constant $F_{p}\left(F_{p}=0.001 \AA\right.$ per pulse period), both independent of frequency (within the range employed in the present work) ${ }^{17}$.

\section{In situ growth monitoring}

Film growth was monitored in situ using a M-88 spectroscopic ellipsometer (J.A. Woollam Inc.) with a rotating analyzer. Ellipsometric angles $\Psi$ and $\Delta$ were acquired every $\sim 2 \AA$ (nominal thickness) at 67 wavelengths in the range $1.6-3.2 \mathrm{eV}$ at an angle of incidence of $65^{\circ}$ from the substrate normal. The acquired data were fitted to a three-phase model consisting of substrate, film and vacuum. The substrate was modeled by a $1 \mathrm{~mm}$ Si slab 
with a top layer of $\mathrm{SiO}_{2}$, the thickness of which was determined by measuring the optical response of the substrate prior to deposition. Reference data for the substrate layers were taken from Herzinger et al. ${ }^{25}$. The optical response of the film was modeled by dispersion and graphical models depending on the point in the film growth evolution, as detailed below.

Films consisting of separated islands were modeled by a single Lorentz oscillator that describes the complex dielectric function, $\tilde{\epsilon}(\omega)$, as a function of the energy of the incoming light, $\omega$, as

$$
\tilde{\epsilon}(\omega)=\epsilon_{\infty}+\frac{\omega_{p}^{2}}{\omega_{0}^{2}-\omega^{2}-i \Gamma \omega} .
$$

In Eq. (6) $\epsilon_{\infty}$ is a constant that accounts for interband transitions at higher energies that are not described by the oscillator, $\omega_{p}$ the plasma frequency, $\omega_{0}$ the resonance energy and $\Gamma$ a damping constant for the electron oscillations. The optical response of the film in this growth regime is described well by Eq. (6) as long as the distance between islands is large enough to not cause additional dipole interactions. Representative ellipsometric spectra (squares) and curves resulting from the best data fit to the three phase model with the film response described by Eq. (6) are shown in Fig. 1 (a). The resulting $\tilde{\epsilon}(\omega)$ of the film is also presented in Fig. 1 (b) with the position of $\omega_{0}$ indicated by the vertically dashed line. By using the calculated $\omega_{0}$ in conjunction with the theory developed by Doremus ${ }^{26}$ one can calculate the substrate surface area covered by $\mathrm{Ag}$ islands, $Q$, at the various conditions through the relationship ${ }^{26}$

$$
\epsilon_{1}\left(\omega_{0}\right)=-\frac{(2+Q) n_{d}^{2}}{1-Q}
$$

Here, $\epsilon_{1}$ is the real part of the corresponding bulk dielectric function of the film (taken from Johnson and Christy ${ }^{27}$ ) and $n_{d}$ is the refractive index of the substrate (taken to be 1.46 for $\mathrm{SiO}_{2}$ ). The relationship may seem simple, but has proven to yield excellent agreement with complementing experimental investigations ${ }^{28}$. By monitoring $\omega_{0}$ during the growth, the evolution of $Q$ was calculated as a function of the nominal thickness. The latter was calculated from the thickness of a continuous film (typically $\sim 250 \AA$ ) as determined by the spectroscopic ellipsometry analysis, assuming a constant growth rate throughout all growth stages. The nominal thickness is expressed in monolayers (ML) where $1 \mathrm{ML}$ corresponds to the lattice spacing between adjacent $\mathrm{Ag}$ (111) planes, which is $2.359 \AA$.

$d_{\text {percol }}$ was obtained using a graphical method developed by Arwin and Aspnes ${ }^{29}$. It utilizes optical features from the underlaying $\mathrm{SiO}_{2}$ layer to concurrently determine $\tilde{\epsilon}(\omega)$ and 


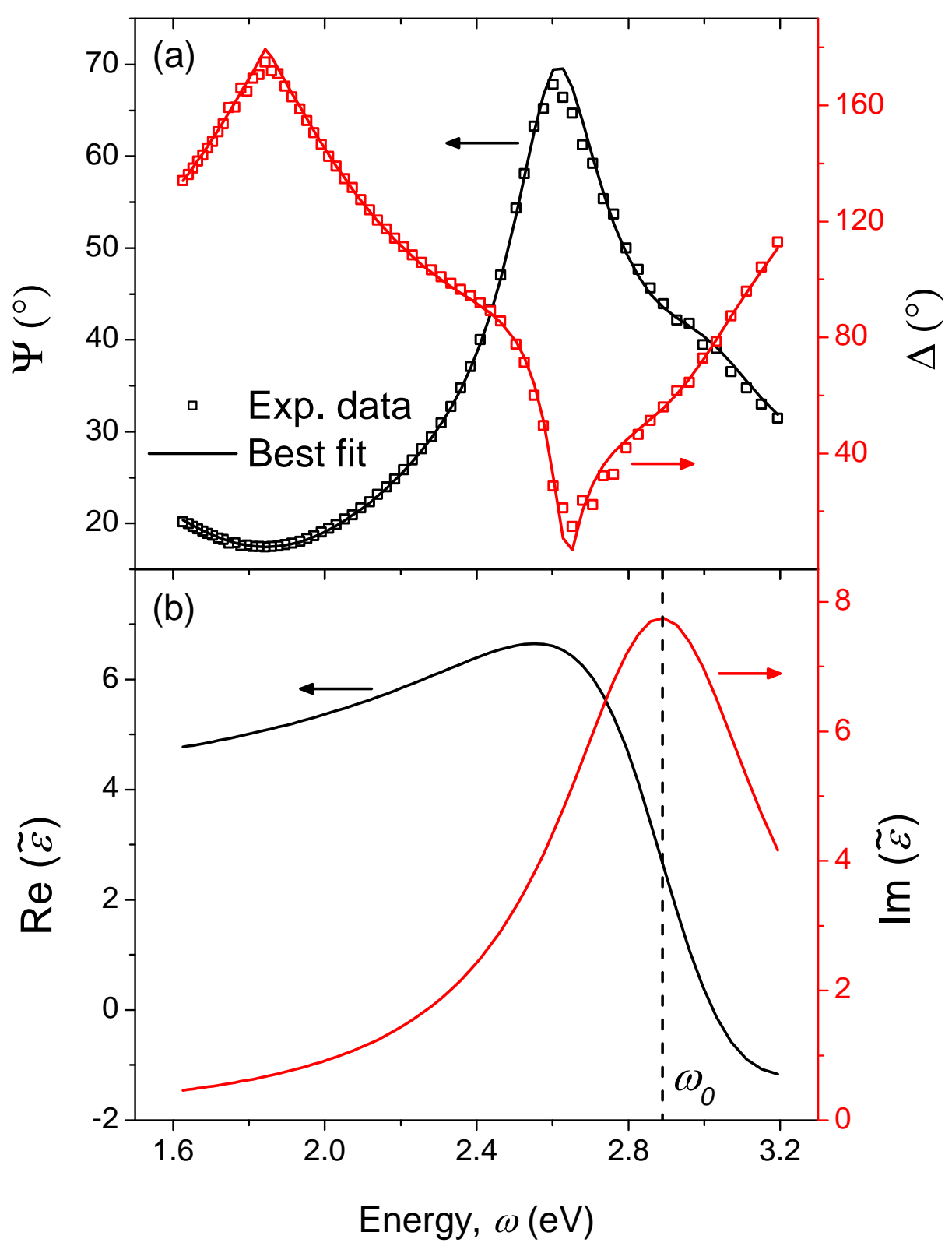

FIG. 1. (a) Measured ellipsometric angles $\Psi$ and $\Delta$ (squares) for a representative Ag film at low nominal thickness together with the best fit (lines) from the three-phase model with the film described by Eq. (6). (b) Imaginary and real part of $\tilde{\epsilon}(\omega)$ for the best fit in (a). The position of $\omega_{0}$ is indicated by a vertically dashed line in (b).

film thickness. The evolution of $\tilde{\epsilon}(\omega)$ together with corresponding nominal film thicknesses using Arwin-Aspnes method are shown in Fig. 2 for a representative Ag film. By noting where the real part of $\tilde{\epsilon}(\omega)$ becomes negative close to the infrared end (low energy) of the spectrum, the nominal thickness where the film starts to show metallic behaviour, $d_{\text {percol }}$, 
can be extracted ${ }^{30}$. This procedure has been shown to be in good agreement with in situ resistivity measurements ${ }^{31}$.

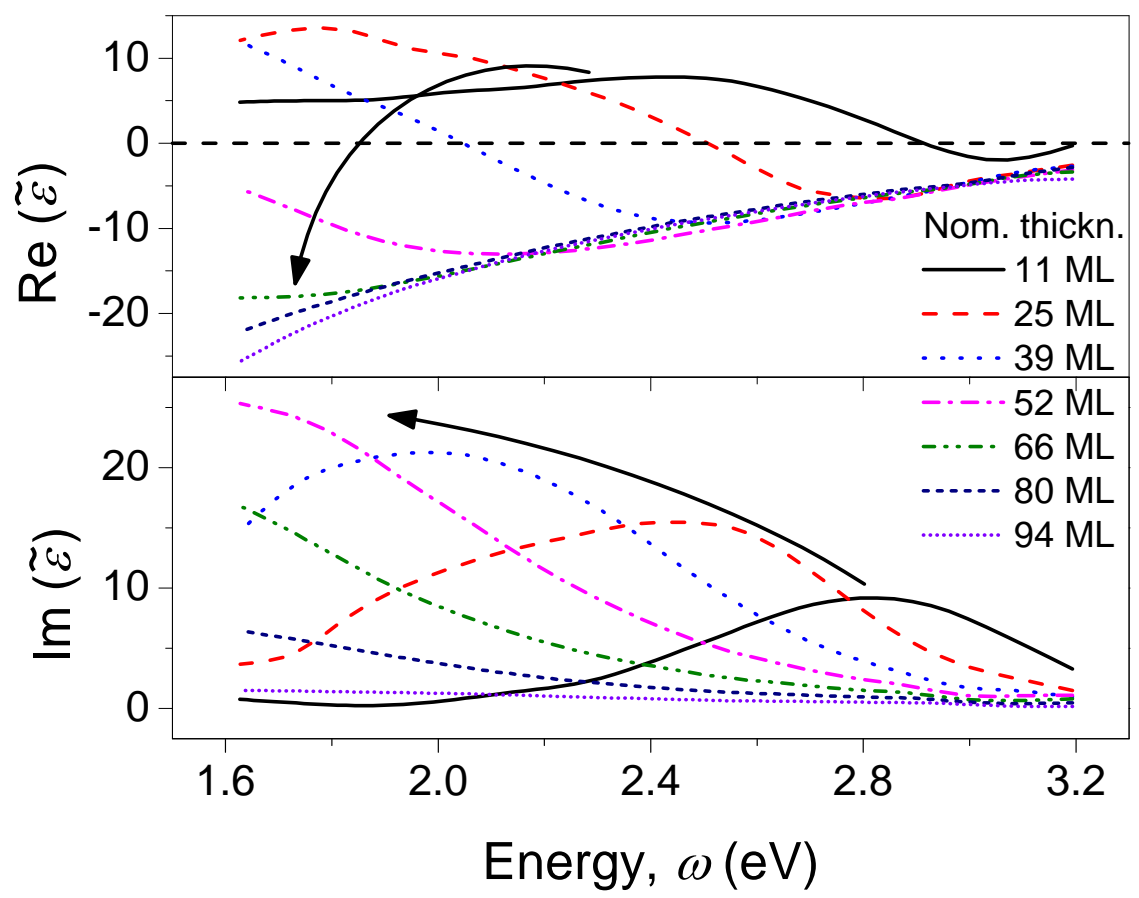

FIG. 2. Evolution of the real and imaginary part of $\tilde{\epsilon}(\omega)$ for a $\mathrm{Ag}$ film grown at $50 \mathrm{~Hz}$ with the corresponding nominal thicknesses, as determined by Arwin-Aspnes method ${ }^{29}$.

The optical response of continuous and close to continuous films were described by the Drude free electron theory, which has been used extensively for ideal metals ${ }^{32}$. In this case, $\tilde{\epsilon}(\omega)$ can be derived from Eq. (6) for $\omega_{0}=0$ and is given by the expression

$$
\tilde{\epsilon}(\omega)=\epsilon_{\infty}-\frac{\omega_{p}^{2}}{\omega^{2}+i \Gamma \omega}
$$

From the best fit parameters the film resistivity $\rho$ was calculated according to ${ }^{33}$

$$
\rho=\frac{\Gamma}{\epsilon_{0} \omega_{p}^{2}},
$$

where $\epsilon_{0}$ is the permittivity of free space. The resistivity was then plotted as a function of the nominal thickness, as seen in Fig. 3. The resistivity experiences a sharp drop as the nominal thickness increases before a steady-state value is reached. The transition to continuous film growth, $d_{\text {cont }}$, was taken at the nominal thickness where a steady-state behaviour was reached ${ }^{34}$. 


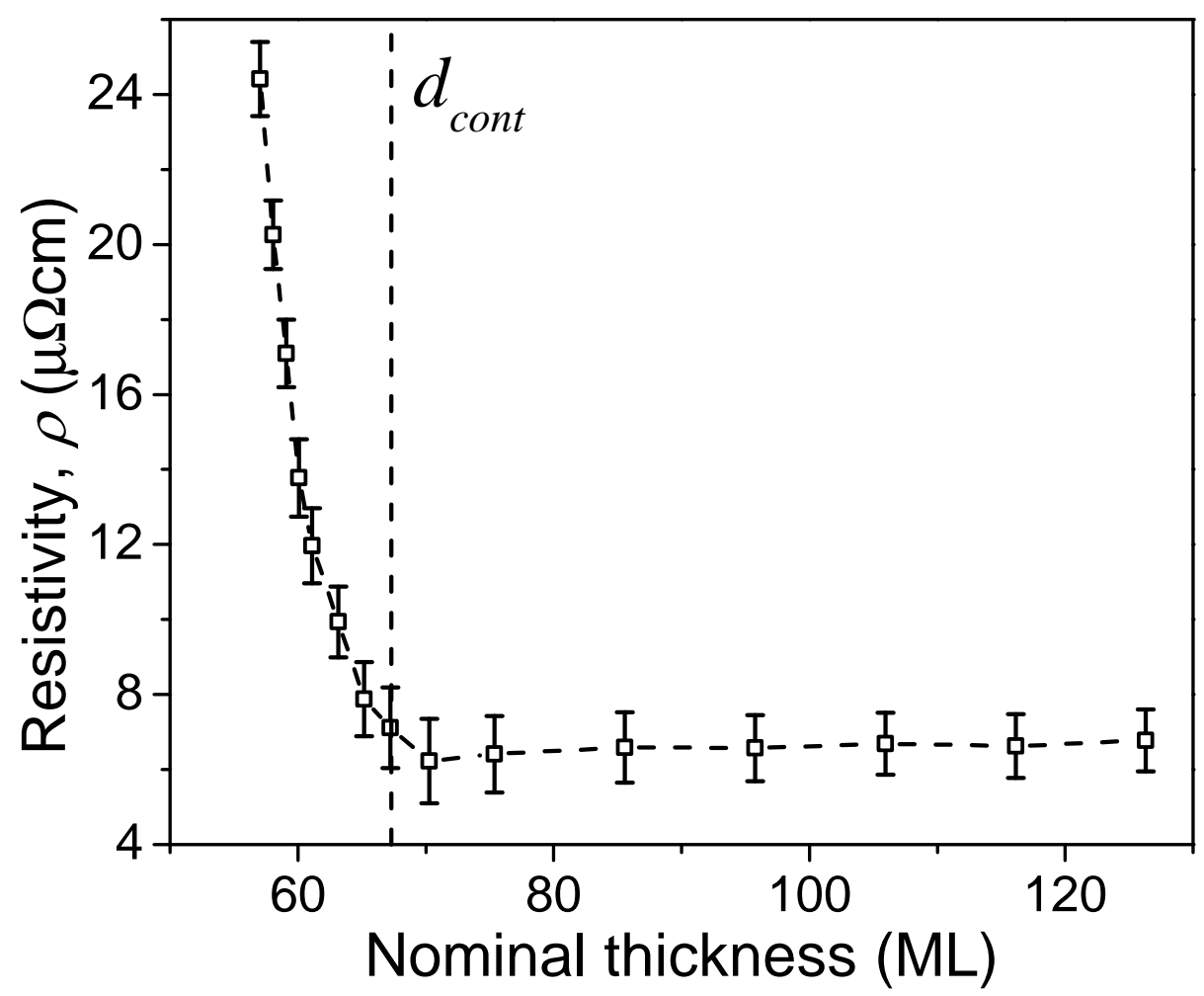

FIG. 3. Evolution of the film resistivity, $\rho$, as a function of the nominal thickness for a Ag film grown at $1000 \mathrm{~Hz}$. The point where $\rho$ reaches a steady-state is used as a measure of $d_{\text {cont }}$, as depicted in the figure.

\section{Ex situ imaging}

Ex situ imaging was performed for selected samples at various characteristic nominal thicknesses by atomic force microscopy (AFM, Nanoscope 3A) working in tapping mode. Images of $1 \times 1 \mu \mathrm{m}$ was acquired at $512 \times 512$ data points, yielding a pixel size of $\sim 2 \mathrm{~nm}$, by using tips with a nominal radius of curvature of $10 \mathrm{~nm}$. Furthermore, the time from deposition interruption to imaging was kept at a minimum in order to minimize any changes of the film morphology that might arise from room temperature annealing.

\section{B. Growth simulations}

KMC simulations were used to study the initial stages of the Volmer-Weber growth up to $d_{\text {elong. }}$. The algorithms used to simulate probability-based atomic processes are welldescribed in textbooks ${ }^{35,36}$, while interactions between islands are based on the theories of 
droplet growth $^{37}$ and thermal grooving ${ }^{38}$. The simulations were set up on a $512 \times 512$ square lattice, onto which atoms were randomly deposited in well-defined pulses. The conditions of total condensation as well as irreversible nucleation were used, with a critical nucleus size of one atom. This implies that no re-evaporation of adatoms or island breakup occurs, and that the smallest stable island consists of two atoms. Adatoms condensed on the substrate are allowed to diffuse with a diffusivity determined by ${ }^{5}$

$$
D=0.25 a^{2} v \exp \left(-E_{D} / k_{B} T\right)
$$

with $a$ the minimum lattice translation distance, $v$ the attempt frequency taken as $5 \cdot 10^{12}$ $\mathrm{s}^{-1}, k_{B}$ the Boltzmann constant and $E_{D}$ the activation barrier for terrace diffusion of the film atoms on the chosen substrate. As atoms get in contact with each other, either by diffusion or deposition, they form a stable island. Similarly, atoms that get in contact with existing islands, either through diffusion or deposition, become incorporated and are placed at positions on the island surface that maintain a hemispherical island shape. In this way, the three-dimensional Volmer-Weber growth geometry is reproduced. The mechanism for coalescence is treated only in pairs of islands, i.e., binary processes, where each island is able to partake in several coalescence pairings simultaneously. $\tau_{\text {coal }}$ is calculated according to Eq. (2), with $R$ as the radius of the smaller island in the pair. If islands grow during an active coalescence process, $\tau_{\text {coal }}$ is recalculated with each island size increment, such that the coalescence process becomes indefinitely delayed if their growth rate is sufficiently high. However, if $\tau_{\text {coal }}$ is reached, the coalescence process is completed by merging the material of the islands into a new island with a center-of-mass located at the size-weighted center-ofmass of the original pair. It should be noted that in the simulations used in the present study two coalescing islands are counted as precisely two islands, even though they are connected with each other, until they fully coalesce into a single island.

To be able to conveniently demark $d_{\text {elong }}$ in the simulations, a ratio $M$ between the total number of islands and the total number of island clusters was tracked throughout the simulations. In this way, $M=2$ signified a point in film growth when each cluster on average consisted of two islands ${ }^{8}$. This is analogous to an elongation of structures on the substrate, since subsequent deposition causes further island impingement to take place, making $M$ a monotonously increasing function in time. The nominal film thickness at this point, $d_{\text {elong}}$, was used to compare different simulations with each other as well as 
to correlate simulations with experimentally determined transition thicknesses $\left(d_{\text {percol }}\right.$ and $\left.d_{\text {cont }}\right)$. The latter correlation can be justified based on previous studies by Carrey and Maurice ${ }^{39}$ who found a linear relationship between $d_{\text {elong }}$ and $d_{\text {percol }}$, both calculated from KMC simulations. This linearity can be understood by the fact that coalescence completion ceases after reaching $d_{\text {elong }}$ and that the decisive process after this point, thus, is island growth that causes islands to impinge on each other and form a continuous network of islands, i.e., reaching $d_{\text {percol }}$. The approach of comparing $d_{\text {elong }}$ data obtained from KMC simulations with experimentally determined $d_{\text {percol }}$ values has previously also been utilized by Warrender and Aziz ${ }^{14}$. Similarly to the number of islands, the number of nucleation, island-island impingement and completed coalescence events, as well as the average island size, were accumulated throughout the simulations. Rates for the three events (nucleation, impingement $\left(1 / \tau_{\text {imp }}\right)$ and coalescence $\left.\left(1 / \tau_{\text {coal }}\right)\right)$ were calculated respectively by taking the difference in number of events for every 0.05 ML of deposit.

For the simulations presented in this paper $f$ was varied between 2 and $6000 \mathrm{~Hz}$ and $t_{\text {on }}$ was fixed at $100 \mu$ s since a major fraction of the experimentally generated pulsed deposition flux is expected to arrive within this time period ${ }^{17,40,41}$. Different values were employed for $E_{D}(0.3$ and $0.4 \mathrm{eV})$ in order to resemble a similar range as the one found in literature ${ }^{42}$, yielding two different $D$ values. $F_{p}$ was fixed at $0.025 \mathrm{ML} /$ pulse for all $f$, and $B$ was chosen to be $500 \mathrm{a}^{4} / \mathrm{s}$. These parameters were chosen in order to facilitate simulations of no more than $\sim 30 \mathrm{ML}$ within a reasonable time frame. They also cause a difference in length scales between simulations and experiments, meaning that the size of islands in the simulations are much smaller than those produced experimentally. Despite this, qualitative trends, such as scaling relations, are unaffected and can be compared with experiments ${ }^{14}$.

\section{RESULTS \& DISCUSSION}

The effect of pulsing frequency on $d_{\text {cont }}$ and $d_{\text {percol }}$ (dots and squares, respectively) are presented in Fig. $4 . d_{\text {cont }}$ takes values from $\sim 94$ to $65 \mathrm{ML}$ as the frequency is varied from 50 to $1000 \mathrm{~Hz}$, while $d_{\text {percol }}$ varies from $\sim 45$ to $33 \mathrm{ML}$ for the same frequencies. Both data sets qualitatively follow the same behaviour, i.e., both transition thicknesses decrease as a power-law from 50 to $400 \mathrm{~Hz}$ with a scaling exponent of -0.18 , after which a steady-state value is reached (i.e., scaling exponent is close to 0 ). These results are qualitatively similar 
to those reported by Warrender and $\mathrm{Aziz}^{14}$ at a growth temperature of $40^{\circ} \mathrm{C}$ who found that $d_{\text {percol }}$ scales with $f$ for pulsed fluxes generated by PLD with an exponent of -0.13 for $2<f<20 \mathrm{~Hz}$ above which a steady-state is reached.

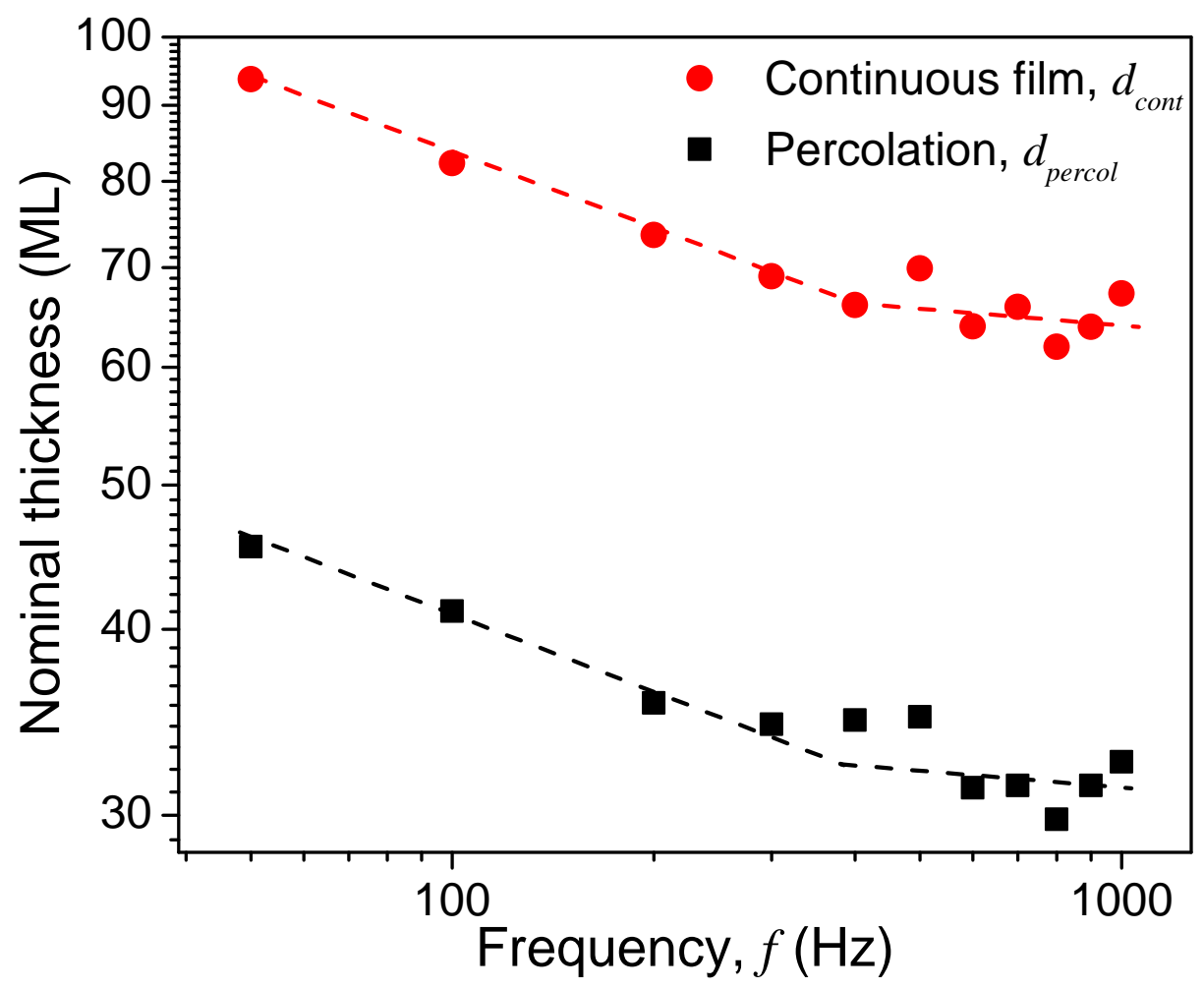

FIG. 4. Effect of pulsing frequency, $f$, on $d_{\text {cont }}$ (dots) and $d_{\text {percol }}$ (squares) for Ag films. The lines are drawn as a guide to the eye.

Fig. 5 displays AFM topographs of Ag films deposited at frequencies of $50 \mathrm{~Hz}$ (Fig. 5 (a)-(d)) and $1000 \mathrm{~Hz}$ (Fig. 5 (e)-(h)). Images were recorded at nominal thicknesses that correspond to $d_{\text {percol }}$ for films deposited at 50 and $1000 \mathrm{~Hz}$ (45 and $33 \mathrm{ML}$, respectively) and $d_{\text {cont }}$ for the same frequencies (94 and $65 \mathrm{ML}$, respectively), as seen in Fig. 5 . The morphological evolution of the film deposited at $50 \mathrm{~Hz}$ (Fig. 5 (a)-(d)) is characteristic of Volmer-Weber growth; isolated islands (a) that grow in size and coalesce forming a continuous island network (b). Subsequent deposition fills in the voids (c) and leads to a continuous film (d). A similar morphological evolution is observed for the film grown at $1000 \mathrm{~Hz}$ (Fig. $5(\mathrm{e})-(\mathrm{h}))$, but in that case the first stage observed is a continuous network of $\mathrm{Ag}$ islands at $d_{\text {percol }}=33 \mathrm{ML}$. Comparison of the surface topography for the two frequencies at their corresponding $d_{\text {percol }}$ (Fig. 5 (b) and (e) for 50 and $1000 \mathrm{~Hz}$, respectively) show that the films 
have similar microstructures. These findings corroborate the results in Fig. 4, i.e., $d_{\text {percol }}$ occurs at different nominal thicknesses depending on the pulsing frequency. The same is valid for $d_{\text {cont }}$ (see Fig. 5 (d) and (g) for 50 and $1000 \mathrm{~Hz}$, respectively). This confirms that the spectroscopic ellipsometry analysis faithfully reproduce results obtained by real space imaging.

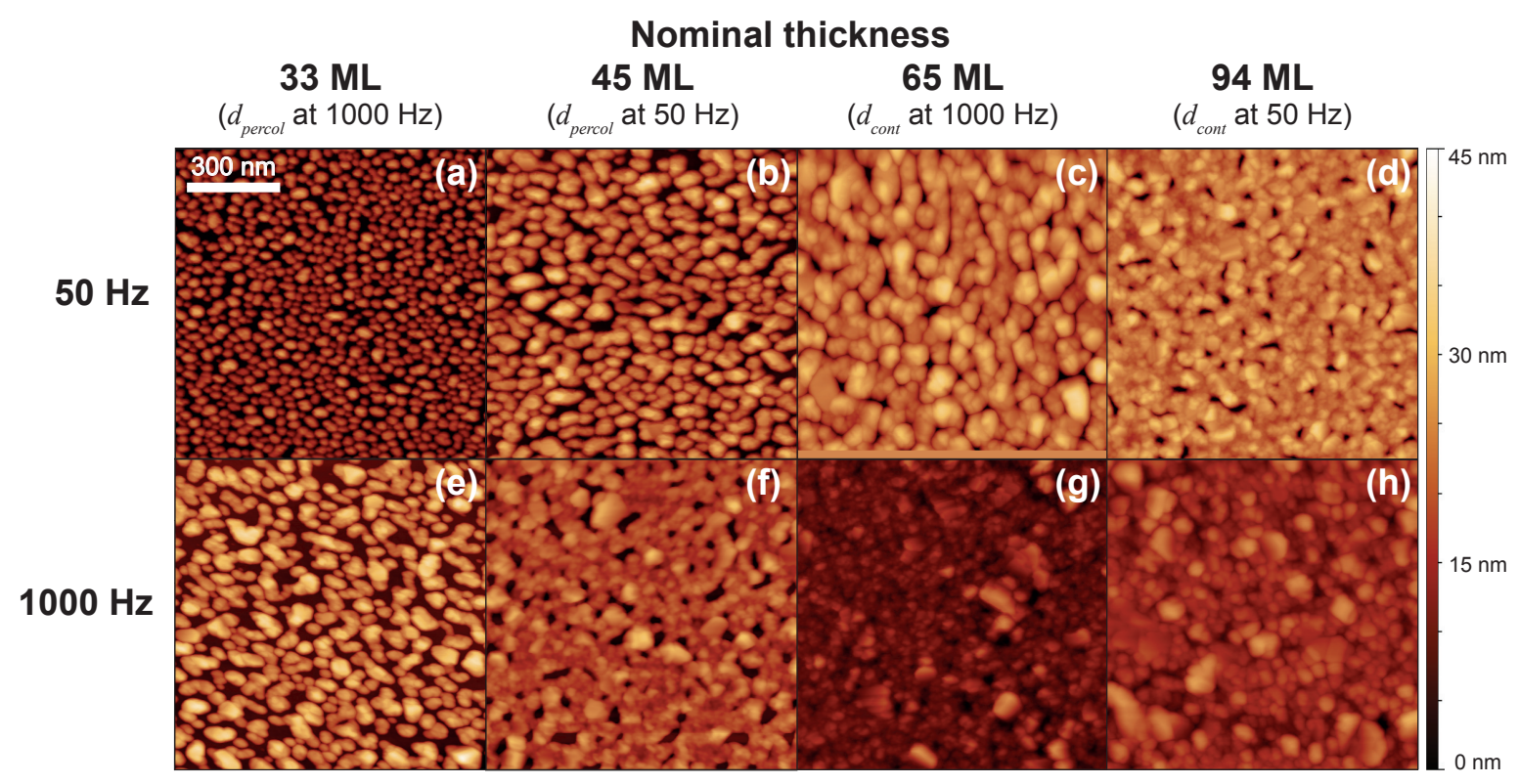

FIG. 5. Microstructural evolution of Ag films at characteristic nominal thicknesses grown at 50 and $1000 \mathrm{~Hz}$ ((a)-(d) and (e)-(h), respectively) as imaged by AFM. Each topograph corresponds to a characteristic nominal thickness from Fig. 4: (a) and (e) $d_{\text {percol }}$ for $1000 \mathrm{~Hz}$, (b) and (f) $d_{\text {percol }}$ for $50 \mathrm{~Hz},(\mathrm{c})$ and (g) $d_{\text {cont }}$ for $1000 \mathrm{~Hz}$, and (d) and (h) $d_{\text {cont }}$ for $50 \mathrm{~Hz}$.

At nominal thicknesses below $d_{\text {percol }}$ the size evolution of the growing islands was studied by calculating the area fraction covered by $\mathrm{Ag}, Q$, for the two extreme pulsing frequencies 50 and $1000 \mathrm{~Hz}$ using Eq. (7). The results are presented in Fig. 6 (triangles and squares for 50 and $1000 \mathrm{~Hz}$, respectively). For the same nominal thickness the film grown at $1000 \mathrm{~Hz}$ covers a larger area on the substrate as compared to the film grown at $50 \mathrm{~Hz}$. The difference in $Q$ between the two frequencies is $\sim 10$ percentage points throughout the monitored range. This indicates a higher island density (more and smaller islands) for the film grown at 1000 $\mathrm{Hz}$, which is consistent with the evolution of $d_{\text {percol }}$ and $d_{\text {cont }}$. The evolution of $Q$ can also be described theoretically by considering a collection of identical and homogeneously spread hemispherical islands that cannot exchange mass with each other. This is achieved by first 
assigning each island to a square area, $A$, on the substrate surface and taking into account the volume, $V=n a A$, of the deposited material, where $n$ is the number of monolayers and $a$ the interplanar spacing for $\mathrm{Ag}(111)$ (i.e., $a=2.359 \AA$ ). Assuming that all deposited material contributes to island growth, the volume of each island is given as $V=2 \pi R^{3} / 3$. Then, $Q$ can be determined as

$$
Q=\frac{\pi R^{2}}{A}=\left(\frac{3 n a}{2}\right)^{\frac{2}{3}}\left(\frac{\pi}{A}\right)^{\frac{1}{3}}
$$

given that $R<A / 2$. Thus, the evolution of $Q$ as function of nominal thickness can be calculated and fitted to experimentally determined values by simply changing the value of $A$. Knowing $A$, the island density is then determined as $1 / A$. This methodology was applied to fit Eq. (11) to the data in Fig. 6. Island densities of $4 \cdot 10^{10}$ (dash-dot) and $1 \cdot 10^{11}$ (dashdash) islands $/ \mathrm{cm}^{2}$ yield very good agreement with the experimental data for the films grown at 50 and $1000 \mathrm{~Hz}$, respectively, as seen in Fig. 6. The calculations thus suggest that 2.5 times more islands are present on the substrate surface for the film grown at $1000 \mathrm{~Hz}$.

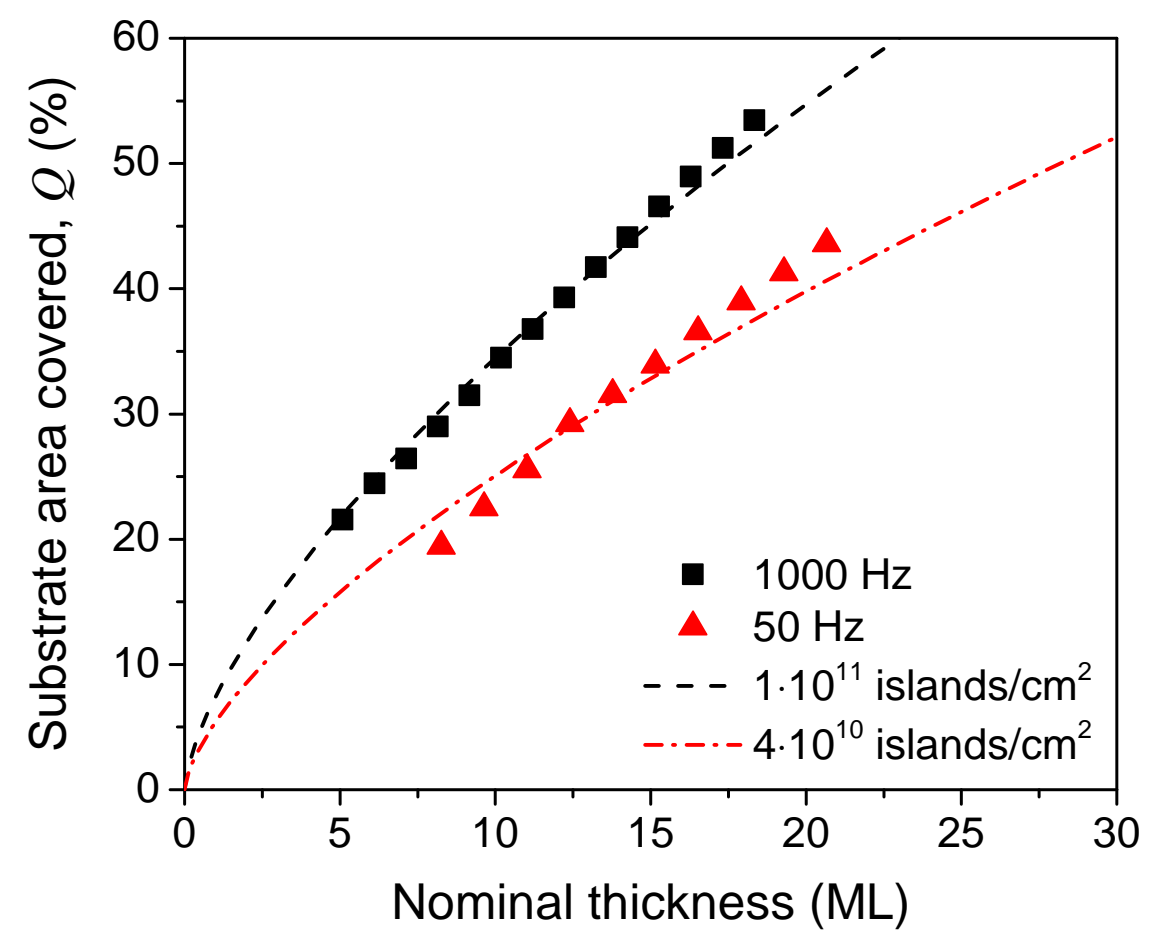

FIG. 6. Evolution of the substrate surface area fraction covered by Ag islands, $Q$, as a function of the nominal thickness for 50 and $1000 \mathrm{~Hz}$ (triangles and squares, respectively). The dashed lines (dash-dot and dash-dash for 50 and $1000 \mathrm{~Hz}$, respectively) are theoretically predicted evolutions using Eq. 11.

Fig. 7 presents $d_{\text {elong }}$ as function of $f$, as calculated from KMC simulations, for two 
different diffusivities, $D=1.1 \cdot 10^{7}$ (squares) and $2.4 \cdot 10^{5}$ (dots) $\mathrm{a}^{2} / \mathrm{s}$ (corresponding to $E_{D}=$ 0.3 and $0.4 \mathrm{eV}$, respectively). $d_{\text {elong }}$ starts to decrease as a power-law with a scaling exponent of -0.33 for both diffusivities as the frequency increases from $2 \mathrm{~Hz}$. This scaling behaviour is consistent with that observed for a continuous flux and is a result of the competition between island growth and coalescence rates ${ }^{8}$. A steady-state value is then reached for frequencies higher than $200 \mathrm{~Hz}$ for $D=1.1 \cdot 10^{7} \mathrm{a}^{2} / \mathrm{s}$, while in the case of $D=2.4 \cdot 10^{5}$ $\mathrm{a}^{2} / \mathrm{s}$ steady-state behaviour is observed above $600 \mathrm{~Hz}$. KMC $d_{\text {elong }}$ data corresponding to the frequency range studied experimentally are also shown separately as an inset in Fig. 7. For this limited frequency range, scaling exponents of -0.17 and -0.25 are obtained for the high and low diffusivity case, respectively, before entering the steady-state regime, i.e., the transition between the two scaling regimes is not sharp. Therefore, by considering the scaling exponent of the KMC $d_{\text {elong }}$ data in this limited frequency range a more relevant comparison with the experimental value of the $d_{\text {percol }}$ and $d_{\text {cont }}$ scaling exponents found from the data presented in Fig. 4 ( -0.18 before entering steady-state after $400 \mathrm{~Hz}$ ) is possible.

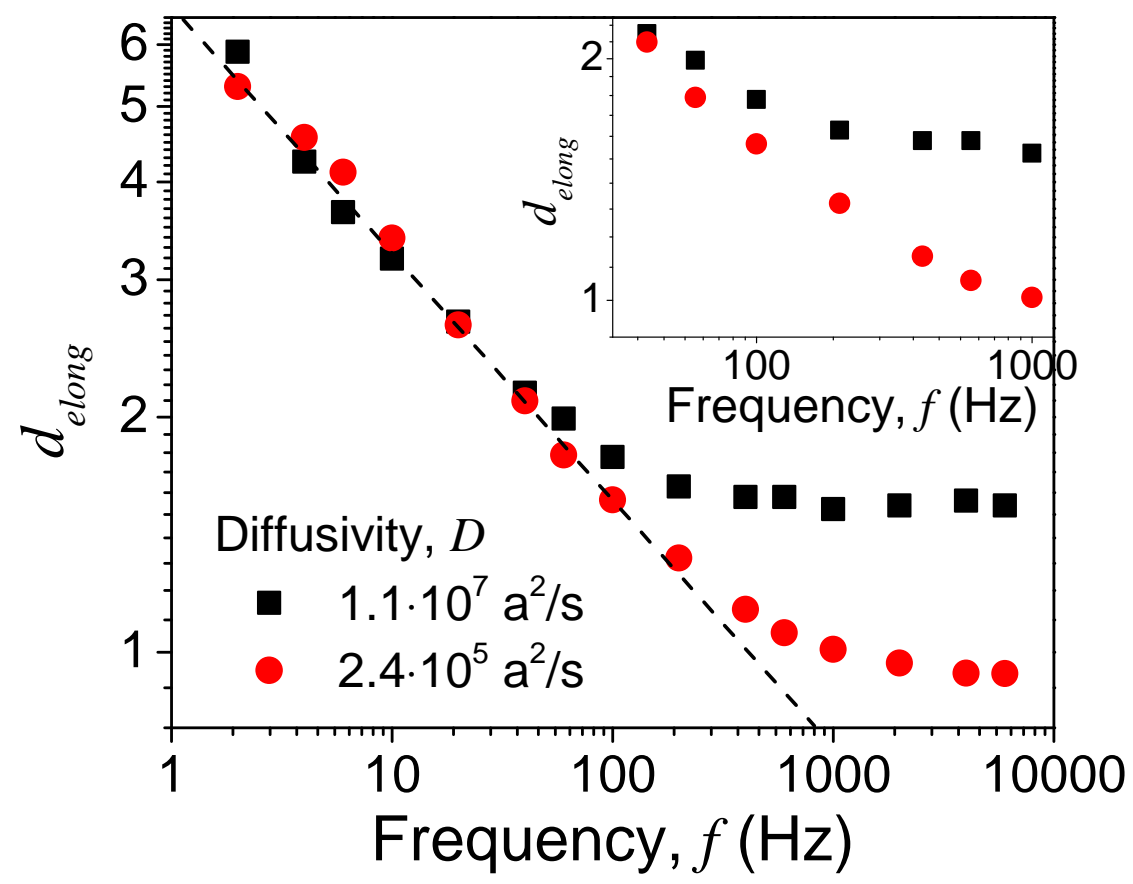

FIG. 7. Elongation transition thickness, $d_{\text {elong }}$, extracted from KMC simulations for two different diffusivities $D=1.1 \cdot 10^{7}$ and $2.4 \cdot 10^{5} \mathrm{a}^{2} / \mathrm{s}$ (squares and dots, respectively), as function of pulsing frequency, $f$. The inset shows the $f$ range studied experimentally. The dashed line is a guide to the eyes. 
It should, however, be noted that the actual value of the experimental scaling exponent can be different than that found in the simulations, since Eq. (2) is only valid at growth temperatures close to the film melting point where surface diffusion and surface tension are isotropic. This is not the case for lower growth temperatures which yield facetted islands where the rate limiting step for coalescence is instead nucleation of new atomic planes on the facets ${ }^{43}$. The latter causes $\tau_{\text {coal }}$ to scale with $R^{\alpha}$, where $\alpha>4,{ }^{43}$ which would favor island growth over coalescence and, hence, lower the characteristic transition thicknesses and lead to a scaling exponent larger than -0.33 (cf. Eq. (3)). It would, however, not invalidate the competition between island growth and island coalescence, and thus the existence of the two scaling regimes. From the above discussion it can be concluded that the scaling behaviour of $d_{\text {percol }}$ and $d_{\text {cont }}$ is in qualitative agreement with that of $d_{\text {elong }}$, which further implies that the scaling behaviour during growth from $d_{\text {elong }}$ to $d_{\text {cont }}$ does not change. Thus, the origin of the effect of time domain on microstructural evolution should be sought in the initial film formation stages. This can be achieved by extracting the island density, nucleation rate, $1 / \tau_{\text {imp }}$ and $1 / \tau_{\text {coal }}$ as a function of nominal thickness from the KMC simulations. This was done for $D=1.1 \cdot 10^{7} \mathrm{a}^{2} / \mathrm{s}$ and frequencies corresponding to the -0.33 scaling regime as well as in the steady-state regime in Fig. 7. As is seen in Fig. 8 (a) the island density is independent of $f$ for low nominal thicknesses $(<0.13 \mathrm{ML})$ after which the island density for different $f$ values starts to deviate from each other. The former is due to the fact that in the transient nucleation regime (i.e., before a steady-state island density is obtained), the island density predominantly scales with the nominal thickness and does not depend strongly on $D$ or other growth conditions ${ }^{5}$. The nucleation rate data (Fig. 8 (b)) show a behaviour that is identical for all frequencies with a sharp increase and a subsequent sharp decrease down to zero (note that all three curves overlap in Fig. 8 (b)). This means that the contribution of the nucleation stage on the resulting island density is the same for all pulsing frequencies. Thus, nucleation is neither responsible for changes in island densities seen in Fig. 8 (a) nor for the scaling behaviour of characteristic transition thicknesses observed in Figs. 4 and 7. Moreover, the constant nucleation rate in Fig. 8 (b) in combination with the fact that $D$, $F_{i}$ and $t_{o n}$ are kept constant and $F_{\text {avg }}$ increases with $f$ suggests that nucleation happens in the fast or the intermediate diffusivity regime with saturation island densities that scale according to Eqs. (4) and (5), respectively. $1 / \tau_{\text {imp }}$ (Fig. 8 (c)) is also independent of $f$, which means that islands grow in the same way up to island impingement irrespective of 


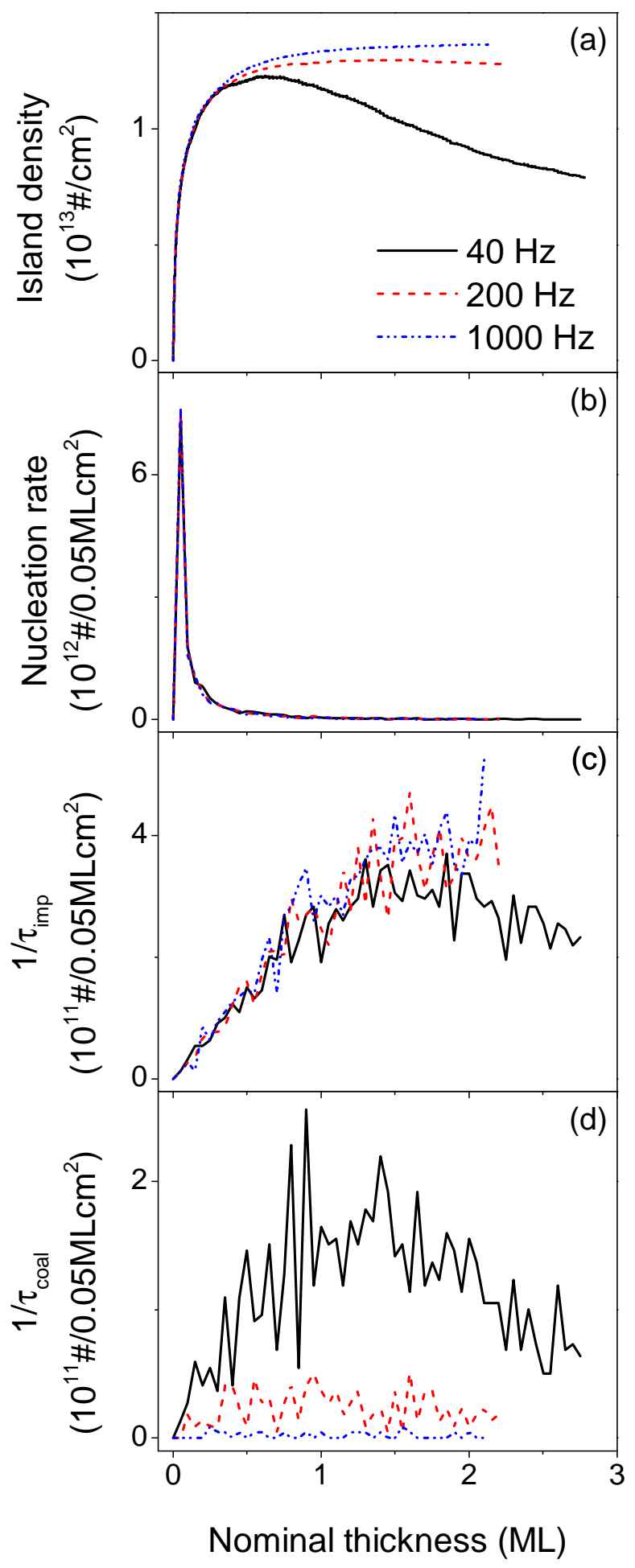

FIG. 8. Evolution of (a) island density, (b) nucleation rate, (c) $1 / \tau_{i m p}$ (island impingement rate) and (d) $1 / \tau_{\text {coal }}$ (coalescence completion rate) as extracted from KMC simulations for various pulsing frequencies at $D=1.1 \cdot 10^{7} \mathrm{a}^{2} / \mathrm{s}$. Note that the curves for all three frequencies overlap in (b). 
$f$ and provides additional evidence for the fact that the time domain of the flux does not affect island nucleation and growth. On the contrary, $1 / \tau_{\text {coal }}$ depends on $f$. For $f=40 \mathrm{~Hz}$ up to $\sim 2 \cdot 10^{11}$ coalescence events per $\mathrm{cm}^{2}$ are completed per $0.05 \mathrm{ML}$ of deposited flux (Fig. 8 (d)). On the other hand, for $f$ values of 200 and $1000 \mathrm{~Hz}$ nearly no coalescence events are completed throughout the simulation. This means that for pulsing frequencies in the -0.33 scaling regime islands of sufficiently small size are given time to complete coalescence before vapor deposition leads to increase of $R$ and thus also $\tau_{\text {coal }}$. Completion of coalescence results in a decrease of island density which can explain the data in Fig. 8 (a) for $f=40 \mathrm{~Hz}$. This in turn shifts the characteristic transitions $\left(d_{\text {cont }}, d_{\text {percol }}\right.$ and $\left.d_{\text {elong }}\right)$ to larger nominal thicknesses when $f$ is decreased since deposition of more material is required for islands to grow sufficiently large causing $1 / \tau_{\text {imp }}$ to exceed $1 / \tau_{\text {coal }}$ (see Figs. 4,5 and 7 ). For $f$ values in the steady-state scaling regime (Figs. 4 and 7 ) island growth dominates, increasing $\tau_{\text {coal }}$ and hindering coalescence from being completed. The latter in combination with the fact that island nucleation and growth do not change with $f$ means that films grow in the same way in the steady-state regime, which explains why the characteristic transition thicknesses $\left(d_{\text {cont }}, d_{\text {percol }}\right.$ and $\left.d_{\text {elong }}\right)$ do not depend on $f$.

The notion of the dominant role of island growth on scaling behaviour is further supported by the data in Fig. 9 where the average island size in number of atoms is plotted against nominal thickness over a wide range of $f$ for $D=1.1 \cdot 10^{7} \mathrm{a}^{2} / \mathrm{s}$. It can be seen that the average island size grows exponentially with nominal thickness for relatively small $f$ values that correspond to the -0.33 scaling regime. This relation becomes practically linear at higher $f$ within the steady-state regime. In general, islands can grow by direct capture of atoms from the vapor flux or by incorporation of diffusing adatoms. In the -0.33 scaling regime, coalescence completion acts as an additional island growth mechanism, both by generating larger islands as well as reducing the number of islands to average over. In the steady-state regime, coalescence completion becomes impeded, which means that islands only grow by direct capture of atoms from the deposition flux and by incorporation of adatoms diffusing on the substrate surface. This, in combination with identical diffusivity conditions and the same nucleation behaviour, causes the average island size evolution to become linear with nominal thickness and increasingly similar for increasing $f$.

Warrender and Aziz ${ }^{14}$ also suggested that coalescence can become impeded at relatively high pulsing frequencies, by observing the existence of a coalescence-free growth regime 


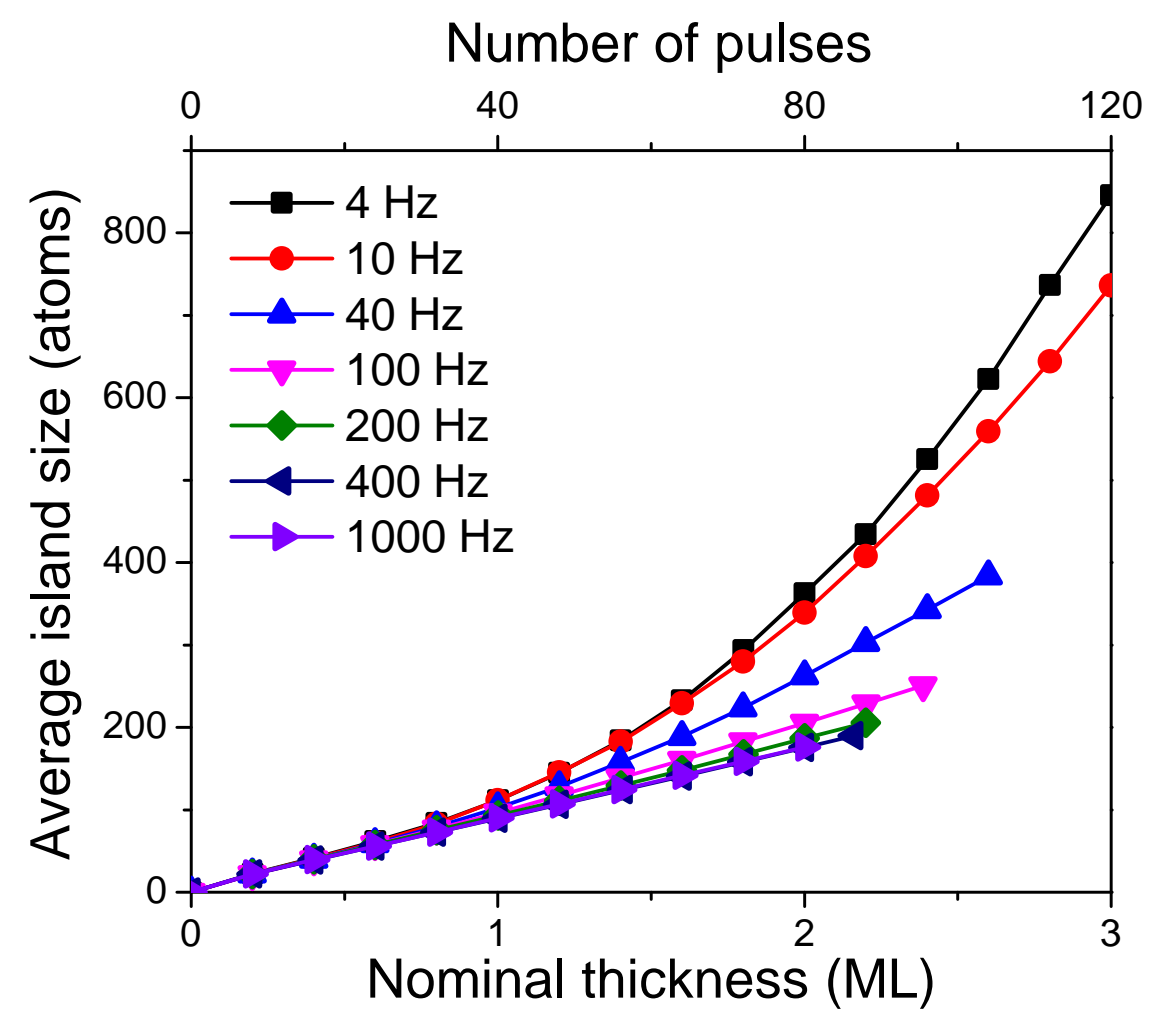

FIG. 9. Average island size in number of atoms for various pulsing frequencies as extracted from the KMC simulations for $D=1.1 \cdot 10^{7} \mathrm{a}^{2} / \mathrm{s}$. Only data for every $0.2 \mathrm{ML}$ are plotted for clarity.

in their KMC data for a case of virtually zero adatom diffusivity $\left(D=10^{-2} \mathrm{a}^{2} / \mathrm{s}\right)$. By having no diffusion, the only island growth mechanism (other than coalescence) is direct capture of atoms from the deposition flux, which would make it difficult for island growth to impede coalescence. The latter argument can be supported by data provided in Ref. ${ }^{14}$ which allow for calculating the average island size at $d_{\text {elong }}$. This can be achieved by using the quantity $\zeta_{\text {elong }}$ that is introduced in Ref. ${ }^{14}$ (see Fig. 3 (d) in Ref. ${ }^{14}$ ), which represents the average coalescence time of all islands that begin coalescing at $d_{\text {elong }}$ divided by the pulse period, $\zeta_{\text {elong }}=\left\langle\tau_{\text {coal }}\right\rangle /(1 / f)$. Using these data we find $\tau_{\text {coal }}$ to be in the range $\sim 0.3-0.5$ s. Then from Eq. (2) and assuming that $R=(3 S / 2 \pi)^{1 / 3}$ for a hemispherical island, where $S$ is the island size in number of atoms, it can be shown that the average island size at $d_{\text {elong }}$ in the coalescence-free growth regime of Warrender and Aziz $^{14}$ is between $\sim 5-7$ atoms. These values are significantly smaller than those shown in Fig. 9 ( 150 atoms) for pulsing frequencies corresponding to the steady-state scaling regime. Hence, no significant island growth occurs in the case of no diffusivity, and the elongation transition is reached 
by nucleation driven island impingement. In addition, in absence of island growth between successive pulses the time domain of the deposition flux (as set by the pulsing frequency) becomes irrelevant for the scaling behaviour. Based on the fact that Ag exhibits considerable diffusivity at room temperature, we argue that the mechanism suggested in the present study (i.e., increase of island size) is more relevant for explaining the scaling behaviour at high pulsing frequencies.

\section{SUMMARY}

We have studied growth evolution of $\mathrm{Ag}$ films deposited from pulsed vapor fluxes on $\mathrm{SiO}_{2}$ all the way from nucleation to the formation of a continuous film. This has been accomplished by combining in situ growth monitoring, ex situ imaging and growth simulations. A pulsing frequency dependence has been observed for characteristic film transitions $\left(d_{\text {elong }}, d_{\text {percol }}\right.$ and $d_{\text {cont }}$ ), where the nominal transition thickness decreases with increasing $f$ up to a certain point where a steady-state behaviour is reached. This behaviour has been demonstrated to be correlated with differences in island sizes and densities for the various $f$ values employed. It has also been shown that the scaling behaviour during growth from $d_{\text {elong }}$ to $d_{\text {cont }}$ does not change significantly, which implies that the film growth evolution is set by the initial growth stages. Growth simulation data suggest that island nucleation and island growth characteristics are independent of the vapor flux time domain. The scaling behavior and hence the microstructural evolution is instead determined by the interplay of $\tau_{\text {coal }}$ and the characteristics of the pulsed vapor flux, as set by $f$. Moreover, we provide evidence that transition to steady-state behaviour is governed by island growth which in turn leads to a growth regime where coalescence is not completed.

\section{ACKNOWLEDGMENTS}

VE, BL and KS should like to acknowledge financial support from Linköping University via the "LiU Research Fellows" program, the Swedish Research Council through contract VR 621-2011-5312 and ÅForsk through the project "Towards Next Generation Energy Saving Windows". The authors would also like to acknowledge Prof. Hans Arwin (Laboratory of Applied Optics, Linköping University) for providing the in situ spectroscopic ellipsometer. 


\section{REFERENCES}

${ }^{1}$ P. M. Martin, Handbook of deposition technologies for films and coatings, 3rd ed. (Elsevier Inc., Oxford, 2010).

${ }^{2}$ M. Ohring, Materials science of thin films, 2nd ed. (Academic Press, San Diego, 2002).

${ }^{3}$ M. A. Garcia, J. Phys. D: Appl. Phys. 44, 283001 (2011).

${ }^{4}$ Y. Shiratsuchi, M. Yamamoto, and S. Bader, Prog. Surf. Sci. 82, 121 (2007).

${ }^{5}$ T. Michely and J. Krug, Islands, mounds and atoms: Patterns and processes in crystal growth far from equilibrium (Springer, Berlin, 2004).

${ }^{6}$ F. A. Nichols and W. W. Mullins, J. Appl. Phys. 36, 1826 (1965).

${ }^{7}$ G. Jeffers, M. A. Dubson, and P. M. Duxbury, J. Appl. Phys. 75, 5016 (1994).

${ }^{8}$ J. Carrey and J.-L. Maurice, Phys. Rev. B 63, 245408 (2001).

${ }^{9}$ P. Jensen and B. Niemeyer, Surf. Sci. 384, L823 (1997).

${ }^{10}$ B. Hinnemann, H. Hinrichsen, and D. E. Wolf, Phys. Rev. Lett. 87, 135701 (2001).

${ }^{11}$ P.-M. Lam, S. Liu, and C. Woo, Phys. Rev. B 66, 045408 (2002).

${ }^{12}$ B. Hinnemann, H. Hinrichsen, and D. E. Wolf, Phys. Rev. E 67, 011602 (2003).

${ }^{13}$ M. Schmid, C. Lenauer, a. Buchsbaum, F. Wimmer, G. Rauchbauer, P. Scheiber, G. Betz, and P. Varga, Phys. Rev. Lett. 103, 076101 (2009).

${ }^{14}$ J. Warrender and M. Aziz, Phys. Rev. B 76, 045414 (2007).

${ }^{15}$ T. N. Hansen, J. Schou, and J. G. Lunney, Appl. Phys. Lett. 72, 1829 (1998).

${ }^{16}$ I. Petrov, P. B. Barna, L. Hultman, and J. E. Greene, J. Vac. Sci. Technol. A 21, S117 (2003).

${ }^{17}$ D. Magnfält, V. Elofsson, G. Abadias, U. Helmersson, and K. Sarakinos, J. Phys. D: Appl. Phys. 46, 215303 (2013).

${ }^{18}$ V. Kouznetsov, K. Macák, J. M. Schneider, U. Helmersson, and I. Petrov, Surf. Coat. Technol. 122, 290 (1999).

${ }^{19}$ U. Helmersson, M. Lattemann, J. Bohlmark, A. P. Ehiasarian, and J. T. Gudmundsson, Thin Solid Films 513, 1 (2006).

${ }^{20}$ J. Alami, S. Bolz, and K. Sarakinos, J. Alloys Compd. 483, 530 (2009).

${ }^{21}$ K. Sarakinos, J. Alami, and S. Konstantinidis, Surf. Coat. Technol. 204, 1661 (2010).

${ }^{22}$ A. Anders, Surf. Coat. Technol. 204, 2864 (2010).

${ }^{23}$ D. Lundin and K. Sarakinos, J. Mater. Res. 27, 780 (2012). 
${ }^{24}$ J. T. Gudmundsson, N. Brenning, D. Lundin, and U. Helmersson, J. Vac. Sci. Technol. A 30, 030801 (2012).

${ }^{25}$ C. M. Herzinger, B. Johs, W. A. McGahan, J. A. Woollam, and W. Paulson, J. Appl. Phys. 83, 3323 (1998).

${ }^{26}$ R. H. Doremus, J. Appl. Phys. 37, 2775 (1966).

${ }^{27}$ P. B. Johnson and R. W. Christy, Phys. Rev. B 6, 4370 (1972).

${ }^{28}$ R. Doremus, Thin Solid Films 326, 205 (1998).

${ }^{29}$ H. Arwin and D. E. Aspnes, Thin Solid Films 113, 101 (1984).

${ }^{30}$ H. Nguyen, I. An, and R. W. Collins, Phys. Rev. B 47, 3947 (1993).

${ }^{31}$ T. W. H. Oates, D. R. McKenzie, and M. M. M. Bilek, Phys. Rev. B 70, 195406 (2004).

${ }^{32} \mathrm{~F}$. Wooten, Optical properties of solids (Academic Press, New York, 1972).

${ }^{33}$ T. W. H. Oates, H. Wormeester, and H. Arwin, Prog. Surf. Sci. 86, 328 (2011).

${ }^{34}$ P. Patsalas and S. Logothetidis, J. Appl. Phys. 93, 989 (2003).

${ }^{35}$ P. Kratzer, in Multiscale Simulation Methods in Molecular Sciences, Vol. 42 (Julich Supercomputing Centre, Julich, 2009) pp. 51-76.

${ }^{36}$ J. D. Erlebacher, in Dynamics of Crystal Surfaces and Interfaces (Plenum Press, New York, 1997) pp. 97-107.

${ }^{37}$ P. Meakin, Reports Prog. Phys. 55, 157 (1992).

${ }^{38}$ W. W. Mullins, J. Appl. Phys. 28, 333 (1957).

${ }^{39}$ J. Carrey and J.-L. Maurice, Phys. Rev. B 65, 205401 (2002).

${ }^{40}$ F. Mitschker, M. Prenzel, J. Benedikt, and A. von Keudell, J. Phys. D: Appl. Phys. 45, 402001 (2012).

${ }^{41}$ W. Breilmann, C. Maszl, J. Benedikt, and A. von Keudell, J. Phys. D: Appl. Phys. 46, 485204 (2013).

${ }^{42}$ H. C. Kim, T. L. Alford, and D. R. Allee, Appl. Phys. Lett. 81, 4287 (2002).

${ }^{43}$ N. Combe, P. Jensen and A. Pimpinelli, Phys. Rev. Lett. 85, 110 (2000). 